\title{
Rashba polarization of bulk continuum states
}

\author{
E. E. Krasovskii ${ }^{1,2,3, *}$ and E. V. Chulkov ${ }^{1,2,4}$ \\ ${ }^{1}$ Departamento de Física de Materiales, Facultad de Ciencias Químicas, Universidad del Pais Vasco/Euskal Herriko Unibertsitatea, \\ Apartado 1072, ES-20080 San Sebastián/Donostia, Basque Country, Spain \\ ${ }^{2}$ Donostia International Physics Center (DIPC), Paseo Manuel de Lardizabal 4, ES-20018 San Sebastián/Donostia, \\ Basque Country, Spain \\ ${ }^{3}$ IKERBASQUE, Basque Foundation for Science, ES-48011 Bilbao, Spain \\ ${ }^{4}$ Centro de Física de Materiales CFM-Materials Physics Center MPC, Centro Mixto CSIC-UPV/EHU, Edificio Korta, \\ Avenida de Tolosa 72, ES-20018 San Sebastián, Spain
}

(Received 5 December 2010; revised manuscript received 20 February 2011; published 1 April 2011)

\begin{abstract}
Spin-orbit coupling is shown to lead to a Rashba-type spin polarization of bulk continuum states at the surface of a nonmagnetic system. A qualitative analysis for a model one-dimensional system is presented, as well as $a b$ initio calculations for (111) surfaces of a number of fcc metals. The effect is interpreted in terms of the reflection of the relativistic Bloch waves from the surface barrier, which leads to a beating of the spin density.

DOI: 10.1103/PhysRevB.83.155401

PACS number(s): 71.70.Ej, 73.20.-r, 71.15.Ap
\end{abstract}

\section{INTRODUCTION}

Owing to its importance for spintronics, the effects of spin splitting at surfaces have attracted much attention in recent years. In nonmagnetic solids the spin splitting is caused by spin-orbit interaction. Especially interesting are crystals with a center of inversion, where the bulk continuum states (of the $\mathbf{k}_{\|}$projected band structure) are doubly Kramers degenerate, whereas discrete surface states are energy split and spin polarized. This is known as the Rashba-Bychkov effect, ${ }^{1}$ caused by the presence of the surface, which breaks the inversion invariance of the system. The spin-orbit splitting of surface states was first observed in angle-resolved photoemission on $\mathrm{Au}(111)^{2}$ and then found for many surfaces of clean high-Z metals, for example, $\mathrm{Bi}(111),{ }^{3,4} \mathrm{Sb}(111),{ }^{5}$ and surface alloys. ${ }^{6,7}$ Similar to the surface states the spin-orbit interaction leads to a Rashba-type splitting of quantum well states. ${ }^{8}$ In general, the main effort has been devoted to studying the Rashba-type polarization of the surface-localized discrete states. ${ }^{9-11}$

It is known, however, that also the electronic structure of the bulk continuum is strongly modified at the surface, giving rise to a charge redistribution and surface resonances. Clearly, the effect of spin-orbit coupling is also expected to be different at the surface and in the bulk, leading to a specific spin polarization of the bulk continuum states in the surface region. Indeed, recently, a spin polarization of the photocurrent from the bulk continuum has been observed in angle-resolved photoemission from $\mathrm{Bi}(111) .{ }^{12}$ The difference from the case of surface states is that the Kramers degeneracy is not lifted for continuum states. However, the effect is again due to the spin-orbit coupling in the bulk and to the presence of the surface. It originates from the potential gradient in the surface perpendicular direction and, thus, can be referred to as the Rashba-type effect.

The aim of the present paper is to give a detailed explanation of the origin of the spin polarization of bulk continuum states at the surface. We present calculations for a simple and instructive model of the crystal surface, in which the crystal potential varies only in the surface perpendicular direction, and generalize the concept of Rashba-type spin polarization to the case of bulk continuum states. Spin polarization is understood in terms of reflection of Bloch states from the surface barrier. We further illustrate this phenomenon by ab initio calculations for (111) surfaces of some fcc metals.

\section{RASHBA MODEL FOR BULK CONTINUUM}

Let us consider the spin structure of bulk continuum states in a nonmagnetic semi-infinite crystal caused by spin-orbit coupling. The bound states are standing waves formed by the incident and reflected Bloch waves. (We use the term "bulk continuum" for the continuum spectrum of both the semi-infinite and the infinite crystal.) First, consider an infinite crystal described by a two-component Schrödinger equation with the Hamiltonian

$$
\hat{H}=\frac{p^{2}}{2 m}+V(\vec{r})+\frac{\hbar}{4 m^{2} c^{2}} \vec{\sigma}[\vec{\nabla} V(\vec{r}) \times \vec{p}] .
$$

In the following we use Rydberg atomic units and denote by $\hat{H}_{0}$ the scalar part $-\Delta+V(\vec{r})$. For a system inhomogeneous only in the surface perpendicular direction (along the $y$ axis; see Fig. 1) the Hamiltonian reduces to

$$
\hat{H}=\hat{H}_{0}+\frac{\alpha^{2}}{4}\left[\left(\begin{array}{cc}
-V_{y}^{\prime} & 0 \\
0 & V_{y}^{\prime}
\end{array}\right) \hat{p}_{x}+\left(\begin{array}{cc}
0 & V_{y}^{\prime} \\
V_{y}^{\prime} & 0
\end{array}\right) \hat{p}_{z}\right],
$$

where $\alpha$ is the fine-structure constant, and $V_{y}^{\prime}$ is the potential gradient in the surface perpendicular direction $y$. Then the Cartesian variables separate, and for a given energy $E$ and surface parallel momentum $\mathbf{k}_{\|}$(let it be along the $x$ axis), the two-component wave function has the form $\Psi(\vec{r})=$ $\Psi(y) \exp \left(i k_{\|} x\right)$, where $\Psi(y)$ is a spinor,

$$
\Psi(y)=\left(\begin{array}{c}
\psi^{\uparrow} \\
\psi^{\downarrow}
\end{array}\right),
$$



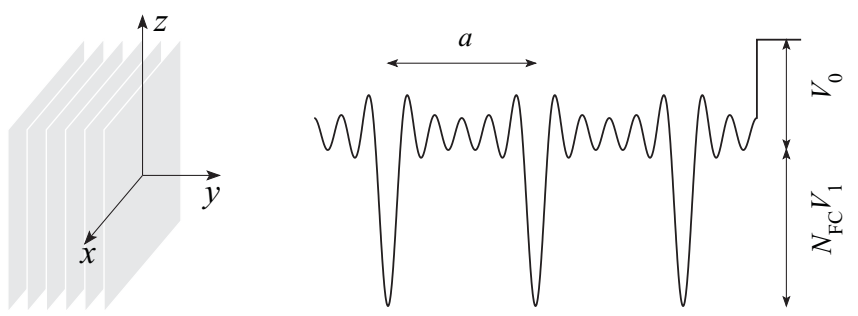

FIG. 1. Coordinate frame and the model one-dimensional potential given by a finite number $N_{\mathrm{FC}}$ of Fourier components of equal amplitude $V_{1}: V(y)=-V_{1} \sum_{n} \cos (2 \pi n y / a+\pi n)$. The potential in vacuum is $V_{0}$. The potential minima simulate atomic layers separated by the lattice constant $a$. Calculations are performed with $a=\pi a_{0}$ and $V_{1}=0.2$ Ry.

with the components $\psi^{\uparrow}$ and $\psi^{\downarrow}$ depending only on the surface perpendicular coordinate $y$. The one-dimensional (1D) Schrödinger equation then reads

$$
\hat{H}_{0}\left(\begin{array}{c}
\psi^{\uparrow} \\
\psi^{\downarrow}
\end{array}\right)+\eta\left(\begin{array}{cc}
-1 & 0 \\
0 & 1
\end{array}\right)\left(\begin{array}{l}
\psi^{\uparrow} \\
\psi^{\downarrow}
\end{array}\right)=E\left(\begin{array}{l}
\psi^{\uparrow} \\
\psi^{\downarrow}
\end{array}\right),
$$

where $\eta=\alpha^{2} V_{y}^{\prime} k_{\|} / 4$. The equations for the two spin projections along the $z$ axis separate, and the problem is reduced to a pair of scalar $1 \mathrm{D}$ equations,

$$
-\psi^{\prime \prime} \pm \eta \psi+V \psi=\left(E-k_{\|}^{2}\right) \psi,
$$

with spin-dependent effective potentials: $v^{\uparrow}=V+\eta$ and $v^{\downarrow}=V-\eta$. For a system with inversion symmetry it is $V(y)=V(-y)$ and $v^{\uparrow}(y)=v^{\downarrow}(-y)$. In the continuum spectrum of the infinite crystal, each of the scalar equations (5) has two propagating solutions $u_{ \pm k}^{\sigma}(y) \exp ( \pm i k y)$ with Bloch vectors $\pm k$. Their periodic parts $u_{ \pm k}^{\sigma}(y)$ are connected by the complex conjugation $u_{-k}^{\sigma}(y)=u_{+k}^{\sigma *}(y)$. Also, the wave functions of opposite spins are simply connected: $u_{+k}^{\downarrow}(y)=$ $u_{-k}^{\uparrow}(-y)$.

If the energy $E$ falls in a forbidden gap, the vectors $k$ are complex, and only one solution per spin (the evanescent wave) is physically meaningful. It gives rise to a discrete eigenstate: the surface state. Because the potentials $v^{\uparrow}$ and $v^{\downarrow}$ are different, the surface states for the two spins occur at different energies, which is known as Rashba splitting of surface states.

For a crystal with inversion symmetry, in the bulk continuum the bands for the two spins are not split, and the eigenstates of the semi-infinite crystal are standing waves (in the surface perpendicular direction). Far from the crystal surface, where the potential is periodic, the wave function is a superposition of the incident and reflected Bloch waves:

$$
\psi^{\sigma}(y)=u^{\sigma}(y) e^{i\left(k y+\phi^{\sigma} / 2\right)}+u^{\sigma *}(y) e^{-i\left(k y+\phi^{\sigma} / 2\right)},
$$

with $u^{\downarrow}(y)=u^{\uparrow *}(-y)$. The phase shifts $\phi$ between the incident and the reflected wave are, in general, different for spin $\uparrow$ and spin $\downarrow$, and they depend on the potential at the surface. For an infinite barrier at the inversion center $y=0$ (of the infinite crystal), it is $\phi^{\downarrow}=-\phi^{\uparrow}$.

The probability density $\rho^{\sigma}$ for each of the spin eigenfunctions defined by $k_{\|}$and $E$ is a sum of the lattice periodic bulk density $\rho^{(\mathrm{b}) \sigma}(y)=2\left|u^{\sigma}(y)\right|^{2}$ and the beating
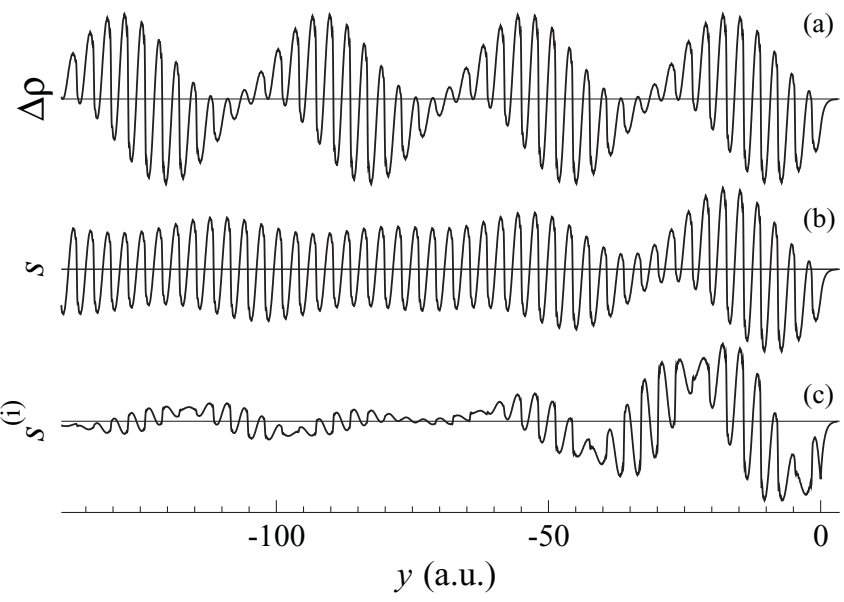

FIG. 2. (a) Spatial distribution of the spin density $\Delta \rho_{k \|}(y, E)$ [see Eq. (6)] associated with a pair of spin eigenfunctions of a semiinfinite crystal (Fig. 1) for $k_{\|}=a_{0}^{-1}$ at $E-k_{\|}^{2}=0.8$ Ry. (b) Spatial distribution of the spin spectral density $s_{k_{\|}}(y, E)$ [see Eq. (7)] at this energy. (c) The beating term $s_{k_{\|}}^{(\mathrm{i})}(y, E)$.

term $\rho^{(\mathrm{i}) \sigma}(y)=2 \operatorname{Re}\left[u^{\sigma}(y) \exp \left(i \phi^{\sigma} / 2+i k y\right)\right]^{2}$ due to the interference between the incident and the reflected Bloch waves. Because $u^{\uparrow}$ and $u^{\downarrow}$ are different, the spin density

$$
\Delta \rho_{k_{\|}}(y, E)=\left|\psi_{k_{\|}}^{\uparrow}(y, E)\right|^{2}-\left|\psi_{k_{\|}}^{\downarrow}(y, E)\right|^{2}
$$

is an oscillating function; see Fig. 2(a). The physically relevant quantity is the spin polarization of the spatially resolved spectral density:

$s_{k_{\|}}(y, E)=\int_{0}^{\pi / a} d k_{y} \Delta \rho_{k_{\|}}\left[y, E\left(k_{\|}, k_{y}\right)\right] \delta\left[E-E\left(k_{\|}, k_{y}\right)\right]$,

where the integration is over the positive $k_{y}$ in the $1 \mathrm{D} \mathrm{BZ}$ in the surface perpendicular direction. The lattice-periodic oscillations of $s_{k_{\|}}(y, E)$ due to

$$
\Delta \rho_{k_{\|}}^{(\mathrm{b})}(y, E)=2\left(\left|u_{k_{\|}}^{\uparrow}(y, E)\right|^{2}-\left|u_{k_{\|}}^{\downarrow}(y, E)\right|^{2}\right)
$$

persist to infinity in the depth of the crystal [Fig. 2(b)], and the beating amplitude upon the integration over $d k_{y}$ decays in the depth as $1 / y$ [Fig. 2(c)], so the net spin spectral density $S\left(k_{\|}, E\right)$ can be obtained by integrating $s_{k_{\|}}^{(\mathrm{i})}(y, E)$ to infinity. For the sake of presentation we define the spin polarization of bulk continuum as $S\left(k_{\|}, E\right)$ divided by the $1 \mathrm{D}$ density of states (DOS) $N\left(k_{\|}, E\right)=\left|d E\left(k_{\|}, k_{y}\right) / d k_{y}\right|^{-1}$ :

$$
\begin{aligned}
p\left(k_{\|}, E\right)= & \frac{1}{N\left(k_{\|}, E\right)} \int_{-\infty}^{+\infty} d y \int_{0}^{\pi / a} d k_{y} \Delta \rho_{k_{\|}}^{(\mathrm{i})}\left[y, E\left(k_{\|}, k_{y}\right)\right] \\
& \times \delta\left[E-E\left(k_{\|}, k_{y}\right)\right] .
\end{aligned}
$$

With the Bloch functions normalized to unit average density the polarization $p$ is measured in units of $a_{0}$. The energy dependence $p(E)$ for the model 1D potential is shown in Fig. 3 . It is seen to depend strongly on the surface barrier. At the same time, the $p(E)$ curves are practically independent of the sharpness of the bulk potential, that is, of the parameter $N_{\mathrm{FC}}$ (see caption to Fig. 1). Far from the band edges the function $p(E)$ changes slowly with energy, and with a sufficiently weak 

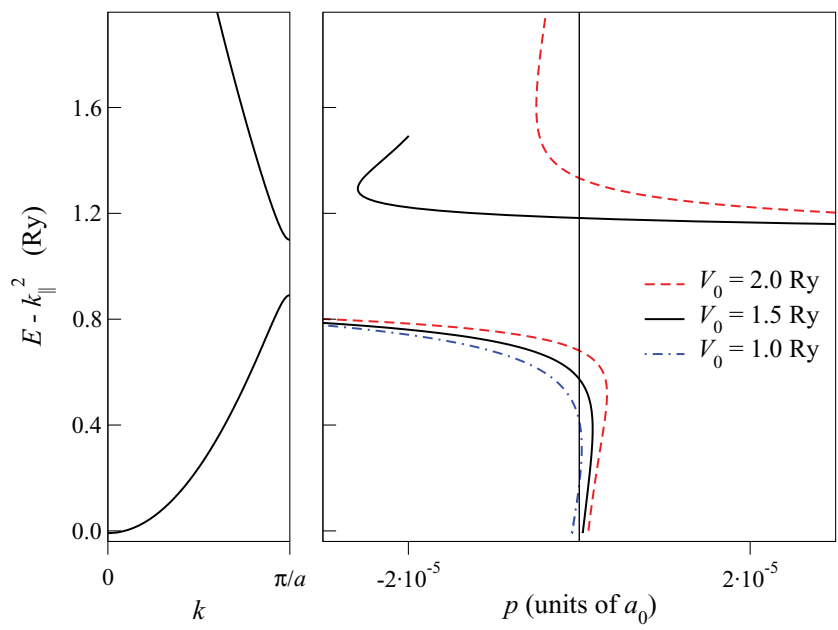

FIG. 3. (Color online) One-dimensional band structure $E(k)$ (left) and spin polarization $p(E)$ of the bulk continuum (right) for the model potential in Fig. 1 with $V_{1}=0.2 \mathrm{Ry}$ and $N_{\mathrm{FC}}=11$. The functions $p(E)$ are shown for three values of the surface barrier: $V_{0}=2 \mathrm{Ry}$ [dashed (red) curve], 1.5 Ry [solid (black) curve], and 1 Ry [dotdashed (blue) curve].

potential $V_{1}$ the polarization in the middle of the band grows linearly with $V_{1}$.

In approaching the band edge the spin polarization grows rapidly and reaches a finite limit at $k=\pi / a$. For a weak crystal potential, $V_{1} \ll(\pi / a)^{2}$, the limit at the zone boundary is $p_{\mathrm{ZB}}=\alpha^{2} \pi^{2} k_{\|} /\left(\sqrt{2} V_{1} a^{2}\right)$, where we have also used that $\alpha^{2} k_{\|} / a \ll 1 \mathrm{Ry}$. (In the limit $V_{1} \rightarrow 0$ the value $p_{\mathrm{ZB}}$ diverges as a result of the normalization to the DOS, which becomes regular as the band gap disappears.)

The above simple model, thus, predicts a finite spin polarization in the continuum spectrum of a semi-infinite crystal, which is spatially localized in the surface region. In contrast to the Rashba effect for surface states, the polarization is not a consequence of the energy splitting of the pure spin states, but originates from a spin-dependent beating between the incident on the surface and reflected Bloch waves. In the next section we consider this effect in realistic materials.

\section{III. (111) SURFACES OF FCC METALS}

To reveal the range of the Rashba effect in the continuum spectrum of realistic crystals and its dependence on the atomic number, we consider (111) surfaces of some noble and late transition metals within an $a b$ initio approach. Instead of a true semi-infinite crystal the surfaces are modeled by thick slabs (19 atomic layers), so the continuum is modeled by a dense set of levels $E_{\lambda \mathbf{k}_{\|}}$. This limits the energy resolution of the spectral density functions, but otherwise this approach correctly yields the observables.

Calculations are performed in a repeated slab geometry with the self-consistent (in the local density approximation) full-potential linear augmented plane-wave method. ${ }^{13}$ The relativistic effects are included within the twocomponent Koelling-Harmon approximation. ${ }^{14,15}$ The local depth-resolved $\mathbf{k}_{\|}$projected DOS is defined as the sum of the densities of spin $\sigma$ [integrated over a surface parallel plane $y=$ const, with $\left.\mathbf{r}=\left(\mathbf{r}_{\|}, y\right)\right]$ over all (discrete) states $\lambda$ with energy $E$ and Bloch vector $\mathbf{k}_{\|}$:

$$
\rho_{\mathbf{k}_{\|}}^{\sigma}(y, E)=\int d \mathbf{r}_{\|} \sum_{\lambda}\left|\psi_{\lambda \mathbf{k}_{\|}}^{\sigma}(\mathbf{r})\right|^{2} \delta\left(E_{\lambda \mathbf{k}_{\|}}-E\right) .
$$

The $\delta$ function is replaced by a Gaussian of $0.5-\mathrm{eV}$ FWHM, which is the energy resolution sufficient for the present purposes; see Figs. 4(a) and 4(b).

Figures 4(a) and 4(b) show the $\mathbf{k}_{\|}$projected DOS along the $\bar{\Gamma} \bar{M}$ line of the surface BZ (SBZ) of $\mathrm{Au}(111)$. The close similarity of the distribution maps calculated from the band structure of the infinite crystal and from the discrete slab levels proves that the structure of the bulk continuum is fairly well reproduced in the slab geometry. The energy-momentum spin distribution shown in Fig. 4(c) reveals spin-polarized bands with a characteristic dispersion different from the dispersion of the DOS features. Thus, in agreement with the simple theory in Sec. II, the continuum spectrum acquires a spin structure, which characterizes the surface of a non-magnetic material. An example of the energy-depth spin density distribution $s(y, E)$ is shown in Fig. 4(d) for $k_{\|}=0.4 \AA^{-1}$. The beating effect is seen to be limited to the outermost four atomic layers, and in the depth of the crystal there remain weak oscillations of the spin density with the lattice periodicity (see Sec. II).

A similar thing also happens in a non-spin-polarized (or nonrelativistic) case, where the reflection of Bloch waves from the surface gives rise to surface resonances, well known in photoemission. From the point of view of spin-resolved photoemission, the relativistic "spin resonances" have the advantage that the surface spin density changes sign, and its spectrum has a richer structure.
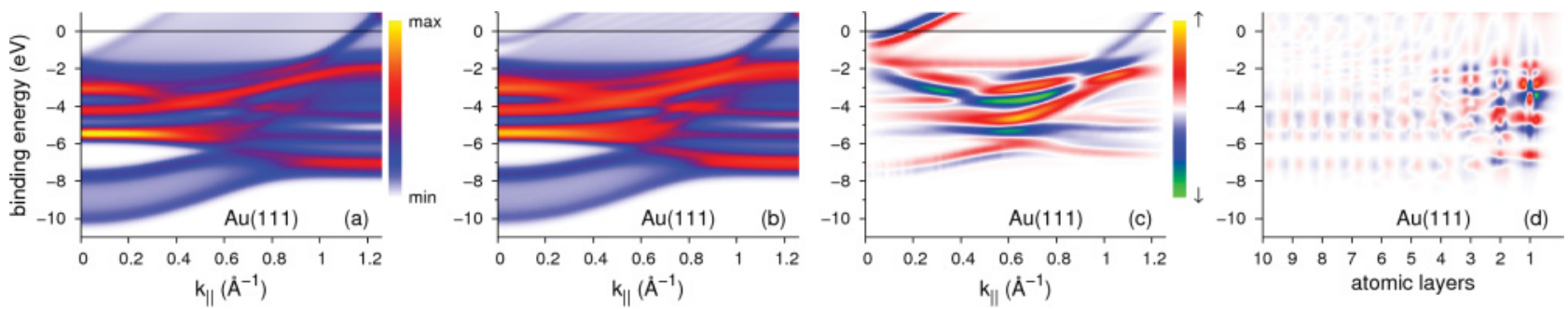

FIG. 4. (Color online) (a, b) Energy-momentum DOS distribution in $\mathrm{Au}(111)$ for $\mathbf{k}_{\|}$along the $\bar{\Gamma} \bar{M}$ line of SBZ obtained (a) from the band structure of the infinite bulk crystal (convoluted with a Gaussian of 0.5-eV FWHM) and (b) from the slab calculation. (c) Energy-momentum distribution of spin polarization along $\bar{\Gamma} \bar{M}$. (d) Local depth-resolved $k_{\|}$projected DOS $\rho_{\mathbf{k} \|}(y, E)$ for $k_{\|}=0.4 \AA^{-1}$. One half of the slab is shown; vacuum is to the right. Tics along the horizontal axis show positions of atomic layers. 

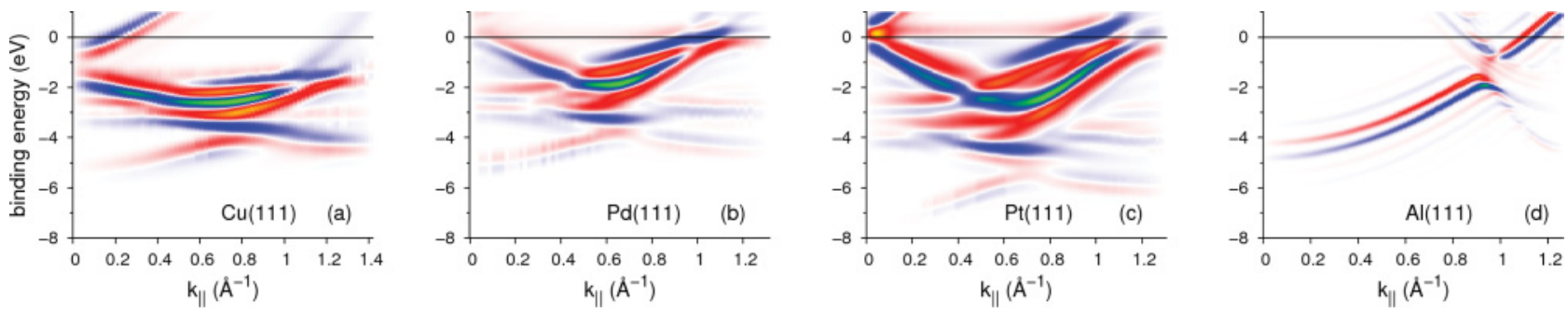

FIG. 5. (Color online) Energy-momentum distribution of spin polarization for $\mathbf{k}_{\|}$along the $\bar{\Gamma} \bar{M}$ line of SBZ in (a) $\mathrm{Cu}(111)$, (b) $\mathrm{Pd}(111)$, (c) $\mathrm{Pt}(111)$, and (d) $\mathrm{Al}(111)$.

Practically the whole effect of spin-orbit coupling comes from the close vicinity of the nucleus (less than $a_{0}$ ), so the magnitude of the surface spin polarization is determined by the atomic number. To illustrate this dependence, we present in Figs. 5(a)-5(c) the polarization maps for $\mathrm{Cu}, \mathrm{Pd}$, and $\mathrm{Pt}$, which have a valence band structure very similar to Au. The $S\left(k_{\|}, E\right)$ maps of the four surfaces are seen to be very similar: the gross features are simply stretched in energy according to the width of the $d$ band. Note that the maxima and minima of the $S\left(k_{\|}, E\right)$ function are well separated in energy: the "splitting" in the continuum spectrum is much larger than the Rashba splitting of the surface states at the same $k_{\|}$. Thus, in photoemission the polarized bands can be detected with a much lower energy resolution than needed to resolve Rashba split surface states, especially in materials composed of lighter atoms. At the same time, the spin sensitivity must be much higher because of the distributed character of $S\left(k_{\|}, E\right)$.

The characteristic magnitude of the effect can be expressed by the integrated absolute value of $S\left(k_{\|}, E\right)$ over the occupied valence band:

$$
M\left(k_{\|}\right)=\int_{\mathrm{VBmin}}^{E_{\mathrm{F}}} d E\left|S\left(k_{\|}, E\right)\right| .
$$

The $M\left(k_{\|}\right)$curves for the four $d$ metals are shown in Fig. 6. On a large scale the characteristic magnitude is seen to grow more rapidly than the atomic number; however, in $\mathrm{Cu}$ it is only four times lower than in $\mathrm{Au}$. In $\mathrm{Bi}(111)$, where the spin polarization in bulk continuum was first observed in photoemission, ${ }^{12}$ the average value of $M$ is around 1 . Thus, we expect this effect to be detectable also in the present materials.

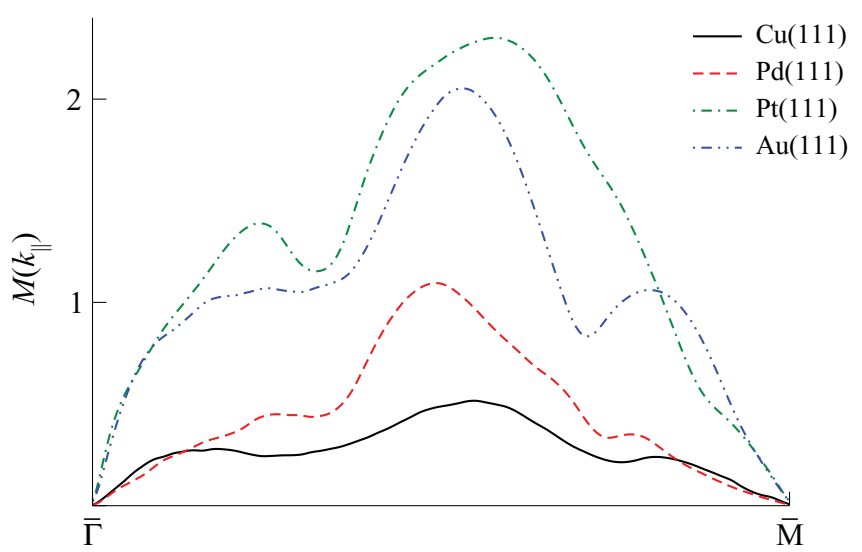

FIG. 6. (Color online) Integrated absolute value of $S\left(k_{\|}, E\right)$ along the $\bar{\Gamma} \bar{M}$ line of the SBZ of (111) surfaces of $\mathrm{Cu}, \mathrm{Pd}, \mathrm{Pt}$, and $\mathrm{Au}$.
An interesting case is provided by the calculation of the $\mathrm{Al}(111)$ film [Fig. 5(d)]. Owing to the high group velocity in the valence band of this simple metal, a 19-layer slab represents a quantum well system rather than a semi-infinite crystal. The quantum well states are given by discrete levels, with the distance greater than $0.5 \mathrm{eV}$ in the energy region shown. Thus, the $0.25-\mathrm{eV}$-wide gap at $\bar{\Gamma}$ at $-4.5 \mathrm{eV}$ in the bulk energy spectrum is not manifested. At the surface of the semi-infinite $\mathrm{Al}(111)$ crystal this gap contains a surface state, which is Rashba split and fully polarized. Because Al is a light atom the splitting is very small, but the polarization of the surface states does not depend on the strength of the spin-orbit coupling. Two of the quantum well states bear the character of the surface state, and with increasing film thickness they will evolve into the surface state. Figure 5(d) shows that just these two states (split by the quantum well confinement) are strongly oppositely polarized, in contrast to the other states, which evolve into bulk states. Thus, the surface state precursor character is reflected in the spin polarization of the film states, which demonstrates that for the quantum well system the surface plays the same role as for the semi-infinite crystal.

\section{CONCLUSIONS}

To summarize, we have presented a theory of the Rashba effect for the continuum spectrum: in a semi-infinite crystal the spin-orbit interaction leads to a surface spin polarization of the continuum states. The local spin density of states exhibits strongly energy-dependent long-range spatial oscillations, which decay in the depth of the crystal. This can be understood as a result of the reflection of the relativistic Bloch waves from the surface barrier, which leads to a beating of the spin density. The developed 1D model demonstrates the polarization to be due to the potential gradient in the surface perpendicular direction and, thus, generalizes the Rashba effect to continuum spectrum.

The effect can be observed in angle-resolved photoemission, as a consequence of the surface sensitivity of this technique. This was recently shown for Bi(111) in Ref. 12 . Our calculations for the metals with localized $d$ bands show that the energy-momentum distribution of the net surface spin has a very rich structure, which reflects special points of the $\mathbf{k}_{\|}$projected band structure. The spectral maxima of opposite spin are well separated in energy, so the effect is expected to be observable also in lighter materials, in which the Rashba splitting of surface states may be within the experimental resolution. 


\section{ACKNOWLEDGMENTS}

The authors gratefully acknowledge fruitful discussions with I. A. Nechaev. The authors acknowledge partial sup- port from the University of the Basque Country (Grant No. GIC07IT36607) and the Spanish Ministerio de Ciencia e Innovación (Grant No. FIS2010-19609-C02-00). *eugene_krasovskii@ehu.es

${ }^{1}$ Y. A. Bychkov and E. I. Rashba, JETP Lett. 39, 78 (1984).

${ }^{2}$ S. LaShell, B. A. McDougall, and E. Jensen, Phys. Rev. Lett. 77, 3419 (1996).

${ }^{3}$ Y. M. Koroteev, G. Bihlmayer, J. E. Gayone, E. V. Chulkov, S. Blügel, P. M. Echenique, and Ph. Hofmann, Phys. Rev. Lett. 93, 046403 (2004).

${ }^{4}$ T. Hirahara, K. Miyamoto, I. Matsuda, T. Kadono, A. Kimura, T. Nagao, G. Bihlmayer, E. V. Chulkov, S. Qiao, K. Shimada, H. Namatame, M. Taniguchi, and S. Hasegawa, Phys. Rev. B 76, 153305 (2007).

${ }^{5}$ K. Sugawara, T. Sato, S. Souma, T. Takahashi, M. Arai, and T. Sasaki, Phys. Rev. Lett. 96, 046411 (2006).

${ }^{6}$ C. R. Ast, J. Henk, A. Ernst, L. Moreschini, M. C. Falub, D. Pacile, P. Bruno, K. Kern, and M. Grioni, Phys. Rev. Lett. 98, 186807 (2007).

${ }^{7}$ H. Bentmann, F. Forster, G. Bihlmayer, E. V. Chulkov, L. Moreschini, M. Grioni, and F. Reinert, Europhys. Lett. 87, 37003 (2009).
${ }^{8}$ J. H. Dil, F. Meier, J. Lobo-Checa, L. Patthey, G. Bihlmayer, and J. Osterwalder, Phys. Rev. Lett. 101, 266802 (2008).

${ }^{9}$ J. I. Pascual, G. Bihlmayer, Yu. M. Koroteev, H.-P. Rust, G. Ceballos, M. Hansmann, K. Horn, E. V. Chulkov, S. Blügel, P. M. Echenique, and Ph. Hofmann, Phys. Rev. Lett. 93, 196802 (2004).

${ }^{10}$ J. H. Dil, J. Phys. Condens. Matter 21, 403001 (2009).

${ }^{11}$ K. Sakamoto, H. Kakuta, K. Sugawara, K. Miyamoto, A. Kimura, T. Kuzumaki, N. Ueno, E. Annese, J. Fujii, A. Kodama, T. Shishidou, H. Namatame, M. Taniguchi, T. Sato, T. Takahashi, and T. Oguchi, Phys. Rev. Lett. 103, 156801 (2009).

${ }^{12}$ A. Kimura, E. E. Krasovskii, R. Nishimura, K. Miyamoto, T. Kadono, K. Kanomaru, E. V. Chulkov, G. Bihlmayer, K. Shimada, H. Namatame, and M. Taniguchi, Phys. Rev. Lett. 105, 076804 (2010).

${ }^{13}$ E. E. Krasovskii, F. Starrost, and W. Schattke, Phys. Rev. B 59, 10504 (1999).

${ }^{14}$ D. D. Koelling and B. N. Harmon, J. Phys. C 10, 3107 (1977).

${ }^{15}$ A. H. MacDonald, W. E. Pickett, and D. D. Koelling, J. Phys. C 13, 2675 (1980). 